\title{
The effect of high- and low-frequency previews and sentential fit on word skipping during reading
}

\author{
Bernhard Angele ${ }^{1}$, Abby Laishley ${ }^{1}$, Keith Rayner ${ }^{2}$, and Simon P. Liversedge ${ }^{3}$ \\ ${ }^{1}$ Psychology Research Centre, Faculty of Science and Technology, Bournemouth University \\ ${ }^{2}$ Department of Psychology, University of California San Diego \\ ${ }^{3}$ School of Psychology, University of Southampton
}

\begin{abstract}
In a previous gaze-contingent boundary experiment, Angele and Rayner (2012) found that readers are likely to skip a word that appears to be the definite article the even when syntactic constraints do not allow for articles to occur in that position. In the present study, we investigated whether the word frequency of the preview of a three-letter target word influences a reader's decision to fixate or skip that word. We found that the word frequency rather than the felicitousness (syntactic fit) of the preview affected how often the upcoming word was skipped. These results indicate that visual information about the upcoming word trumps information from the sentence context when it comes to making a skipping decision. Skipping parafoveal instances of the therefore may simply be an extreme case of skipping high-frequency words.
\end{abstract}

In order to allocate their gaze efficiently, readers have to anticipate how much information they will need about upcoming (that is, not yet fixated) words. There are two possible sources for information about upcoming words: First, readers can use parafoveal vision to preprocess words before they are fixated (McConkie \& Rayner, 1975; Rayner, 1975). However, there is debate about the extent and the quality of the parafoveal information that is available when the oculomotor system makes a decision about where to fixate next and when to make the next saccade (see Liversedge \& Findlay, 2000; Rayner, 1998, 2009; Rayner \& Liversedge, 2011; Schotter, Angele, \& Rayner, 2012 for reviews) ${ }^{1}$. Second, readers can also use the preceding sentence context to predict/guess (at an unconscious level) the identity of the upcoming words (Balota, Pollatsek, \& Rayner, 1985; Bicknell \& Levy, 2010; Ehrlich \& Rayner, 1981; Levy, Bicknell, Slattery, \& Rayner, 2009; Rayner \& Well, 1996). However, such predictions/guesses may not always be correct and may require input from high-level sentence integration processes which might not be available early enough to influence oculomotor decisions. The question we will consider is to what extent readers use parafoveal information and sentence context information when they make a skipping decision.

Correspondence to: Bernhard Angele, Psychology Research Centre, Faculty of Science and Technology, Bournemouth University, Talbot Campus, Fern Barrow, Poole BH12 5BB, United Kingdom, bangele@bournemouth.ac.uk.

${ }^{1} \mathrm{An}$ extreme position on this question would hold that skipping is almost entirely explainable by oculomotor factors, including word length (for an example, see Vitu, O'Reagan, Inhoff, \& Topolski, 1995). However, this fails to explain how word frequency and predictability can have an effect on word skipping as detailed below. 
In determining the relative importance of parafoveal information compared to predictability from the sentence context, the phenomenon of word skipping is particularly interesting: if a word is intentionally skipped, this can be taken to imply that it has been parafoveally processed to the point where it no longer needs to be fixated. This assumption is explicitly implemented in serial attention shift models of eye-movement control during reading such as the E-Z Reader model (Reichle, Pollatsek, Fisher, \& Rayner, 1998; Reichle, Warren, \& McConnell, 2009), according to which a word is never intentionally skipped unless it has been parafoveally identified. This view derives from the assumption that words are processed one at a time and in the correct order (but not necessarily fixated in that order). Word skipping in E- $Z$ Reader occurs because readers start preprocessing the upcoming word parafoveally whenever they have identified the currently fixated word but their oculomotor system is not yet ready to perform a saccade to the next word. If the upcoming word is very easy to process, it is possible that it too is identified before the saccadic program has completed. In this case, the current saccadic program is cancelled and a saccade to the second word to the right—-that is, a skipping saccade—is prepared ${ }^{2}$.

A different approach to word skipping - also stressing the importance of parafoveal information - is made by processing gradient models such as the SWIFT model of eyemovement control (Engbert, Nuthmann, Richter, \& Kliegl, 2005), which assume that multiple words can be processed in parallel and therefore allows for word skipping even if a word has not been fully identified. Specifically, SWIFT proposes a dynamic field of activations with one activation value for each word in a sentence. The maximum activation value of each word is determined by its word frequency. Initially, the further a word has been processed, the higher its activation value is (predictable words rise in activation more slowly than unpredictable words). Once the activation of a word reaches its maximum, further processing lowers the activation value until it is back at zero at which point the word is assumed to be fully identified. In SWIFT, saccades are triggered at random intervals (with a possible delay due to foveal word difficulty). The probability of a word becoming the target of the next saccade is directly proportional to its activation level relative to the activation levels of the other words in the field. This means that any word that has been processed to any degree and that is not yet fully identified can be a potential saccade target in SWIFT. Because of this, skipping the upcoming word is possible as soon as the word after it has accumulated some activation. For example, if the upcoming word has a relative activation of .7 and the word to its right has a relative activation of .2, SWIFT will skip the upcoming word in $20 \%$ of simulations (and fixate it in $70 \%$ of simulations[JA1][BA2]).

Importantly, both E-Z Reader and SWIFT allow for an influence of word predictability from the sentence context on skipping probability. However, neither model is very clear on how the oculomotor system estimates predictability. In particular, both models assume that, in order for predictability to play a role in word identification, the word in question must have received at least some parafoveal preprocessing - simply guessing a word without any parafoveal input does not occur according to either model. Finally, both the SWIFT and E-Z Reader models can also account for accidental word skipping due to oculomotor error.

\footnotetext{
${ }^{2}$ In rare cases, the oculomotor error built into E-Z Reader can lead to accidental skipping of a word. However, in this case, skipping the word would not be considered intentional.
} 
Much of the research on word skipping has focused on those words that are skipped most often during reading, such as the definite article the in English or the plural definite article les in French. O'Regan (1979) and Gautier, Le Gargasson, and O'Regan (2000) found that les was skipped more often than other three-letter words even when it was not predictable from the prior context. Angele and Rayner (2012) investigated this "automatic" the-skipping phenomenon further by using the gaze-contingent boundary paradigm (Rayner, 1975) to present readers with infelicitous (syntactically illegal) previews of the article the. This was done by having subjects read sentences containing three-letter target verbs (e.g. ate). In the critical condition, while fixating to the left of the target word, the parafoveal preview for the target verb was replaced with the article the. This ensured that all the-previews were infelicitous in the sentence context. There were two additional conditions, one in which the preview was correct (control condition) and a random letter nonword preview condition.

After crossing the boundary, readers always saw the correct word. Angele and Rayner found that readers skipped nearly $50 \%$ of the words that appeared as the, even when the sentence context did not syntactically allow an article in the target word position. This skipping rate was comparable with that calculated for felicitous occurrences of the in other positions in the sentences, suggesting that, in accordance with Gautier et al.'s results, skipping of articles is not strongly influenced by constraints of the preceding sentence context. In other words, Angele and Rayner's results seem to indicate that word skipping is mainly influenced by parafoveal processing. However, it is not clear whether article previews are skipped more often than correct verb previews because of their higher word frequency alone, or whether the lower fixation probability is specific to the article the (and perhaps a small number of other common, short function words).

In order to investigate this important theoretical question further, we used a paradigm that was quite similar to the one used by Angele and Rayner (2012). Specifically, we used threeletter target words ( $\operatorname{dog}$ in the sentence "The excitable dog was ready to go for his walk"), the previews of which could either be correct and compatible with the sentence context (identical control condition, $d o g$ ), a random letter string (fze), or a higher- or lowerfrequency three-letter word that did not fit in the sentence context (dim). According to the CELEX corpus (accessed using the $N$-Watch software by Davis (2005), the low frequency targets had a mean word frequency of $10(\mathrm{SD}=14)$, while the high frequency words had a mean word frequency of 1177 ( $\mathrm{SD}=3110$ ). The mean difference in frequency within each pair was 1168 ( $\mathrm{SD}=3108$; see Table A2 in the Appendix for details). It is important to note that, in the above example, presenting an adjective preview instead of the target word (which was a noun) caused a syntactic violation. This was the case in the majority of our stimuli (see the Method section for details). Due to their limited horizontal extent, such short words should be skipped quite often in reading (Hautala, Hyönä, \& Aro, 2011; Rayner \& McConkie, 1976). On the other hand, high frequency words are skipped more often than low frequency words (White, 2008). As a consequence, we expected there to be a number of potential influences on fixation probability and fixation times.

First, the sentence context is very likely to have a strong influence on all eye-movement measures. However, it is important to note that the main effect of sentence frame is not readily interpretable as the pre- and post-target words necessarily differed between sentence 
frames. Even on the target word, the effect of the context might be influenced by spillover effects from the previous words. Consequently, in addition to the main analysis across sentence frames, we will present separate analyses for those sentence frames in which the target was a high-frequency word (and the dissimilar previews could either be a random letter string or a lower frequency word) and those sentence frames in which the target was a low-frequency word (and the dissimilar previews could be a random letter string or a higher frequency word).Within sentence frames, the context was constant across all preview conditions.

Second, the parafoveal preview should have a clear effect on the processing of the target word. Strong preview benefit effects on the target word were predicted, that is, shorter fixation times when the target word preview was identical to the target word and longer fixation times when it was dissimilar. We also anticipated that these effects might spill over onto the post-target word.

Finally, we expected the parafoveal preview to have an immediate effect on eye movement behavior before fixating the target word: If the preview is a non-word, subjects should be more likely to fixate the target word. The presence of a non-word in the parafovea may also lead to longer fixation times on the pre-target word (such an effect of parafoveal orthographic information on ongoing processing as evidenced by the duration of the current fixation is known as an orthographic parafoveal-on-foveal effect; see Schotter et al., 2012). For those conditions in which the preview is a word (that is, the identical and alternative preview conditions), the probability of skipping the target word could be affected by the frequency of the preview and by the fit between preview and sentence context. If readers only take the frequency of the upcoming word into account, the frequency preview should have a direct influence on whether the target word is skipped or fixated independent of the sentence context, with high-frequency previews leading to higher skipping rates than lowfrequency previews. Alternatively, if readers take the context into account when making a skipping decision, they should be less likely to skip a syntactically invalid preview than a syntactically valid preview. Of course, target word skipping might be affected by both preview word frequency and contextual fit at the same time. Additionally, based on previous studies (Kennedy, 1998; Kennedy, Murray, \& Boissiere, 2004; Kennedy \& Pynte, 2005; Kennedy, Pynte, \& Ducrot, 2002; Kliegl, Nuthmann, \& Engbert, 2006), a low-frequency word in the parafovea might also lead to longer fixation times on the pre-target word than a high-frequency word (a lexical parafoveal-on-foveal effect). In more general theoretical terms, the outcome of this experiment will tell us more about the importance of predictive linguistic processing during reading.

\section{Method}

\section{Subjects}

Thirty-five students from the University of Southampton participated in the experiment for course credit. All were native English speakers with normal/corrected-to-normal vision and no diagnosed reading difficulties. 


\section{Apparatus}

Eye-movements were monitored every millisecond using an SR Research Eyelink 1000 eyetracker. Viewing was binocular, though eye movement data were only collected from the right eye. Sentence stimuli were displayed on a computer monitor with a refresh rate of 150 $\mathrm{Hz}^{3}$. Viewing distance was $73 \mathrm{~cm}$, with 3 characters equaling $1^{\circ}$ of visual angle. A videogame controller was used by subjects to end each trial and respond to comprehension questions.

\section{Materials}

We assembled a list of 120 pairs of three-letter high- and low-frequency words; members of each pair differed in word frequency. Furthermore, we constructed 240 sentence frames (see Appendix for details). Each sentence frame was compatible with either the high or the low frequency word of a pair, but not with the other word of that pair. For most of the word pairs and sentence frames, this incompatibility was syntactic (144 out of 240), but, due to the difficulty inherent in finding word pair/sentence frame combinations resulting in true syntactic anomalies, about one third of the word pair/sentence frame combinations instead had semantic incompatibilities (It was an emotional day/dew for all of the family members, 96 out of 240). Some of the target word pairs also contained content words (e.g. can/cow). We will present a number of supplementary analyses aimed at determining whether these differences between items had an impact on the effect of the preview manipulation at the end of the next section. Each subject read only one of the two sentence frame versions, resulting in an equal number of high-to-low and low-to-high frequency manipulations (see Figure 1 for an example).

To summarize, there were three preview conditions: the preview word was either identical to the target word (dog/dog; dim/dim), a dissimilar random letter-string (fze/dog; fz.j/dim), or the infelicitous lower/higher frequency alternative word ( $\mathrm{dim} / \mathrm{dog} ; \mathrm{dog} / \mathrm{dim})$. Each subject read a total of 120 sentences.

\section{Procedure}

Subjects were asked to read each sentence for comprehension. A chin/forehead rest was used to minimize participant head movements. Initial three-point calibrations were carried out until error was $<0.3^{\circ}$ and re-calibrations were completed as needed. The entire session lasted approximately 45 minutes ${ }^{4}$.

\section{Results and Discussion}

We computed a number of standard eye movement measures (Rayner, 1998, 2009) on the pre-target word (e.g. excitable), the target word (dog), and the post-target word (was). Specifically, we computed two early processing time measures, first fixation duration (FFD; mean duration of the first fixation on a word) and gaze duration (GD; the sum of all fixations on a word before leaving it), both calculated only for words that were not initially

\footnotetext{
${ }^{3}$ At a refresh rate of $150 \mathrm{~Hz}$, the display changes took an average of $3 \mathrm{~ms}$ and a maximum of $6 \mathrm{~ms}$ to be completed after they were initiated. As a consequence, after removing those trials with very late display changes (see Results and Discussion section), the average display change finished less than $2 \mathrm{~ms}$ after fixation onset.
} 
skipped. Most importantly, we computed the probability of skipping the target word as well as the probability of making a regression out of the target word. The latter measure only showed significant effects on the post-target word. We therefore do not report the results from the regression probability analyses in the target and the pre-target word regions. Finally, we also calculated two later processing measures, go-past time (Go-past; the sum of all the fixations on a word and any regressive fixations before moving to the right of the target word) and total time (TT; the sum of all fixations on a word during a trial). Table 1a through 3a show the means and standard deviations for each condition and each of the dependent variables.

In the present study, $28.6 \%$ of the display changes completed more than $10 \mathrm{~ms}$ after the onset of the subsequent fixation. In this case, we discarded the corresponding trial from the analysis. This was done because previous research (Slattery, Angele, \& Rayner, 2011) indicated that display changes delayed by more than $10 \mathrm{~ms}$ cause a change in eye-movement behavior even when subjects are not aware of them. Furthermore, if a fixation was shorter than $80 \mathrm{~ms}$ and located within one character space (11 pixels) of another fixation, it was merged into that fixation, otherwise it was deleted. Fixations with durations that deviated from a subject's mean by more than two standard deviations were deleted as well (around $5 \%$ of the data). All subjects answered at least $85 \%$ of the comprehension questions correctly.

We expected that the frequent skipping of the three-letter target words and exclusion of delayed display changes would lead to unequal cell sizes, which would make the use of ANOVAs to analyze the data difficult. Thus, we used linear mixed models (LMM) with the target word preview condition (identical vs. alternative vs. dissimilar) and the sentence frame used (high frequency target word vs. low frequency target word) as well as their interaction as fixed effects; additionally, the model contained random intercepts and random slopes for preview condition and sentence frame condition (Baayen, Davidson, \& Bates, $2008)^{5}$. For the preview condition, we used successive differences contrasts, comparing the identical with the alternative and the alternative with the dissimilar condition. Since the sentence frames differed in many ways, interpreting the main effect of sentence frame is not possible. However, the interaction of sentence frame with preview is still interesting,

\footnotetext{
${ }^{4}$ There is evidence that awareness of display changes can influence some eye-movement measures (White, Rayner, \& Liversedge, 2005). Out of 53 subjects that were originally tested, the data of 18 subjects who noted display changes on their own without prompting or noticed more than 5 changes were excluded from the analysis because of this (leaving a total of 35 subjects included in the analysis). An analysis of the data from the 18 discarded subjects showed few differences in terms of the effects pattern compared to the subjects in the main analysis; however, a number of effects failed to reach significance, likely due to power limitations. The skipping probability effects that did not reach significance for the discarded subjects were the effect of preview (both contrasts) on the probability of fixating the pre-target word, the difference between the dissimilar and the alternative conditions in terms of the probability of skipping the target word as well as its interaction with sentence frame, and the effect of the interaction between the difference between the dissimilar and the alternative preview and the sentence frame on the probability of skipping the post-target word. The fixation time effects that did not reach significance for the discarded subjects were the interaction between preview and sentence frame on FFD on the pre-target word, the difference between the dissimilar and the alternative preview on FFD and GD on the target word, the interaction between preview and sentence frame on GD on the target word, the effect of sentence frame on FFD on the post-target word, on GD on the target word, and on go-past time on the post-target word, and, finally, the difference between the identical and alternative preview conditions on GD and go-past time on the post-target word. There was only one effect that was significant for the discarded subjects but not for the included subjects, namely an interaction between preview and sentence frame on GD on the post-target word $(b=-76.82, \mathrm{SE}=26.19, t=-2.93)$.

${ }^{5}$ Random slopes for the interaction term could not be included, as the majority of the models no longer converged in this case. A small minority of models did not converge even with the restricted random effects specification described above. In this case, we report a more restricted model including random slopes only for the preview effect.
} 
especially on the target word. In order to further investigate cases where this interaction reached significance, we performed separate analyses for each of the two sets of sentence frames. We used the lmer function from the lme4 package (Bates, Maechler, Bolker, \& Walker, 2013) within the R Environment for Statistical Computing (R Development Core Team, 2013) to fit the LMMs. For each factor (preview word, sentence frame, and their interaction), we report regression coefficients ( $b$ ), standard errors, and $t$-values. For binomial dependent variables such as fixation and regression probabilities, we report regression coefficients, standard errors, and $z$-values from generalized LMMs using a logit-link. It is not clear how to determine the degrees of freedom for the $t$-statistics estimated by the LMMs, making it difficult to estimate $p$-values (Baayen et al., 2008). However, since our analyses contain a large number of subjects and items and only a few fixed and random effects are estimated, we can assume that the distribution of the $t$-values estimated by the LMMs approximates the normal distribution. We therefore used the two-tailed criterion $|t| \geq$ 1.96 which corresponds to a significance test at the 5\% $a$-level. The $z$-values from the generalized LMMs can be interpreted in exactly the same way. Table $1 \mathrm{~b}$ through $3 \mathrm{~b}$ (sentence frames with high-frequency target word) and 1c through $3 \mathrm{c}$ (sentence frames with low-frequency target word) show the LMM results, although the coefficient estimates and statistics for significant effects are also repeated in the text.

\section{Early processing measures: Skipping probability}

Pre-target word-The probability of skipping the pre-target word was influenced by the preview condition; readers were significantly less likely to skip the pre-target word in the alternative condition than in the identical condition $(b=.45, \mathrm{SE}=.18, z=2.49)$. Readers were also less likely to skip the pre-target word in the dissimilar preview condition than in the alternative preview condition $(b=-.44, \mathrm{SE}=.19, z=-2.3)$. These effects, which are similar to effects observed by Angele and Rayner (2012), were unexpected and it is not quite clear what causes them. Perhaps readers are on some occasions able to detect an anomaly arising from the preview manipulation on the target word while fixating two words to the left of it (see Rayner, Angele, Schotter, \& Bicknell (2013) for discussion), though a fair amount of prior research indicates this is unlikely (see Rayner, 1998, 2009 for reviews). Consistent with our last point, note, however, that skipping of this region only occurred on a minority of trials (between 11 and 17\%) - in the majority of trials, readers either do not notice the parafoveal violation or do not react to it until they reach the pre-target word.

Target word-Both the difference in skipping probability between the identical and alternative conditions $(b=.52, \mathrm{SE}=.21, z=2.5)$ and the difference between the alternative and dissimilar conditions $(b=-.61, \mathrm{SE}=.21, z=-2.91)$ were modulated by sentence frame. Specifically, sentence frames with high-frequency targets showed a small, and, likely due to lack of power, only marginally significant difference between the alternative and the identical condition, with the target word being less likely to be skipped in the alternative than in the identical condition $(b=-.29, \mathrm{SE}=.17, z=-1.69)$, and barely any difference at all between the dissimilar and alternative conditions $(b=.00, \mathrm{SE}=.18, z=-.02)$. In contrast, sentence frames with low-frequency targets showed a small difference in the opposite direction between the alternative and the identical conditions, with the target being skipped less often in the identical than in the dissimilar condition $(b=.25, \mathrm{SE}=.15, z=$ 
$-1.64)$, and a very strong difference between the dissimilar and the alternative conditions, with the dissimilar condition resulting in many more target fixations than the alternative condition $(b=-.62, \mathrm{SE}=.15, z=-4.17)$. This interaction suggests that, overall, readers were more likely to skip a target word with a high-frequency preview (the identical preview for high-frequency target sentence frames and the alternative preview for low-frequency target sentence frames) than a target word with a low-frequency preview (the alternative preview for high-frequency target sentence frames and the identical preview for lowfrequency target sentence frames), confirming our prediction.

Post-target word-There was a significant interaction between sentence frame and preview on skipping probability on the post-target word. The difference in skipping probability between the alternative and the dissimilar preview conditions was modulated by sentence frame $(b=-.46, \mathrm{SE}=.23, t=-2.02)$. The separate analyses by sentence frame showed that, for high-frequency target sentence frames, there was a marginally significant difference between the alternative and the dissimilar preview conditions, with the dissimilar preview leading to more skips of the post-target word $(b=-.33, \mathrm{SE}=.18, z=-1.86)$. For low-frequency target sentence frames, on the other hand, this effect was completely absent $(z=.9)^{6}$. In summary, skipping of the post-target word was not strongly affected by the preview manipulation.

\section{Early processing measures: Fixation time measures}

Pre-target word-There were no significant effects of preview, with the exception of a significant interaction between the preview contrast between the identical and the alternative preview and sentence frame in FFD $(b=-14.18, \mathrm{SE}=5.73, t=-2.48)$. However, it is unclear whether this effect can be interpreted ${ }^{7}$. There were no significant effects on other early fixation time measures on the pre-target word (all $t \mathrm{~s}<1.96$ ).

Target word-In the early fixation time measures we found evidence of a standard preview benefit effect (Rayner, 1975): There was a main effect of preview in FFD and GD indicating that fixation times on the target word were shortest in the identical condition compared to the alternative condition (FFD: $b=15.53, \mathrm{SE}=4.94, t=3.14$; GD: $b=19.91$, $\mathrm{SE}=7.61, t=2.62)$. Furthermore, FFD and GD were shorter in the alternative condition than in the dissimilar condition (FFD: $b=13.23, \mathrm{SE}=4.93, t=2.68$, GD: $b=17.34, \mathrm{SE}=$ $6.86, t=2.53$ ), suggesting that there was a cost of having a dissimilar, random letter preview that exceeded the cost of having a preview that was simply a different word. On GD, this effect differed between sentence frames (Interaction term: $b=-25.47, \mathrm{SE}=12.59, t=$ -2.02). Specifically, there was a strong difference between the alternative and the dissimilar

\footnotetext{
${ }^{6}$ There was an overall effect of preview on the probability of making a regression out of the post-target word, with more regressions in the alternative preview condition than in the identical preview condition $(b=.58, \mathrm{SE}=.19, z=3.11)$. This effect was not modulated by sentence frame and suggests that having had a parafoveal preview of a target word (or a dissimilar, random letter nonword) that does not fit in the later sentence context does disrupt further processing to some degree. Even though readers have seen the correct target word at this point, the preview information still seems to have some effect on their processing, possibly at the integration level. ${ }^{7}$ We are very cautious in interpreting this interaction effect, which is limited to FFD, for three reasons: (1) in order to detect the syntactic violation caused by the mismatch of sentence frame and preview, readers would have to have fully identified the preview and integrated it into the sentence frame before actually fixating the target word and (2) even if they did this, it is not clear why fixations would then be shorter in the syntactic violation condition rather than longer. Furthermore, (3) it is unclear why the syntactic violation would not also be detected in the high-frequency sentence frames as well.
} 
condition in sentence frames with a high-frequency target $(b=29.12, \mathrm{SE}=8.43, t=3.46)$ and virtually no difference in sentence frames with a low-frequency target $(\mathrm{b}=4.44, \mathrm{SE}=$ $10.95, t=.4)$. Apparently, processing a low-frequency target word was much more dependent on a correct preview than processing a high-frequency target word. The dissimilar preview proved disruptive in both cases.

Post-target word-There was a spill-over effect of the target word manipulation. Specifically, GD on the post-target were significantly longer in the alternative preview condition compared to the identical condition (GD: $b=22.69, \mathrm{SE}=7.68, t=2.95$ ). This effect did not differ between sentence frames $(t<1.96)$.

\section{Late processing measures}

Pre-target word-There was a main effect of preview on total viewing time in those sentence frames with a high-frequency target word, with a significant difference in TT between the identical high-frequency target previews and the alternative low-frequency word previews $(b=26.35, \mathrm{SE}=11.44, t=2.3)$. There also was a significant interaction between preview (alternative vs. identical contrast) and sentence frame $(b=-35.65, \mathrm{SE}=$ $16.89, t=-2.11$ ). Separate analyses by sentence frame (see Table 1c) showed a significant difference between the alternative and the identical conditions in sentence frames with a high-frequency target word $(b=46.99, \mathrm{SE}=15.11, t=3.11)$, but not in those with a lowfrequency target word $(b=7.66, \mathrm{SE}=16.14, t=.21)$. Overall, preview effects for TT on the pre-target word are most likely due to readers re-reading the pre-target word after having received a dissimilar preview. The fact that such an effect only reached significance for the sentence frames with a high-frequency target word may again indicate that processing was disrupted more when readers were expecting a high-frequency word but receive a dissimilar preview compared to the situation in which readers were expecting a low-frequency word and then receive a high-frequency or random letter preview. Of course, the preceding sentence context may have influenced the effect of a dissimilar preview in other ways as well.

Target word-The preview benefit effect we found on the earlier measures persisted in the late measures, with longer go-past times and TT in the alternative than in the identical condition (go-past: $b=46.29, \mathrm{SE}=13.2, t=3.51$; TT: $b=28.23, \mathrm{SE}=8.71, t=3.24$ ).

Post-target word-We found spillover effects on go-past time and TT (go-past: $b=$ 64.87, $\mathrm{SE}=14.9, t=4.35$; TT: $b=28.67, \mathrm{SE}=9.81, t=2.92$ ), with go-past times and TTs being longer in the alternative preview condition than in the identical condition. Again, this effect was not modulated by sentence frame $(b=-2.75, \mathrm{SE}=18.41, z=-.15)$.

\section{Supplementary analyses}

Syntactic vs. semantic preview violations-As described in the Method section, about one third of the sentence frame/preview word combinations resulted in a semantic rather than syntactic violation (e.g. They looked at the afternoon (sky/cut) and admired the clouds is a semantic preview violation as opposed to the syntactically illegal preview violation in She accidentally (cut/sky) herself while making a collage, where the first word 
in parentheses is the target word and the second word in parentheses is the alternative preview). In order to determine whether the observed effects differed by type of violation, we performed analyses with an additional factor denoting whether the violation in the alternative preview condition was syntactic (coded as 0 ) or semantic (coded as 1 , see Appendix for details). There was no significant interaction effect on skipping probability between violation type and preview and no significant three-way interaction between preview, sentence frame, and violation type (all $z<1.65$ ), suggesting that the effect we observed did not differ between violation types. There were a small number of significant interaction effects on fixation time measures. Specifically, there was a significant interaction between violation type and the comparison between the identical and the alternative previews in FFD and GD on the post-target word (FFD: $\mathrm{b}=-16.67, \mathrm{SE}=8.24, t=-2.02$; GD: $b=-31.87, \mathrm{SE}=14.7, t=-2.17$ ) when the alternative preview constituted a syntactic violation, suggesting that the spillover effect from having had an alternative as opposed to an identical preview of the target word was only present when the violation had been of a syntactic nature. There was also a significant interaction between violation type and the comparison between the alternative and the dissimilar previews in GD and go-past time on the target word (GD: $b=-28.42, \mathrm{SE}=12.97, t=-2.192$; Go-past: $b=-64.15, \mathrm{SE}=24.67, t$ $=-2.60$ ), indicating that, in contrast to the main analysis, there was a difference in preview benefit between the alternative and the dissimilar conditions, but only when the preview violation was of a syntactic nature. However, as the present study was not designed to investigate the effect of preview violation types and our analyses may not have enough power to detect subtle effects of violation type, further research will be necessary in order to confirm that, for the purposes of making a skipping decision, semantic and syntactic preview violations are equivalent (that is, that neither type has an effect on skipping).

Content vs. function word targets and previews-Another factor that may be important in the present study is the use of both content and function words as targets and alternative previews (e.g. The old man across the street (has/hem) a very bad cold vs. My grandmother told me she would (hem/has) that dress for me, where the first word in parentheses is the target word and the second word in parentheses is the alternative preview). The advantage of using function words as targets and previews is that this is likely to result in a strong syntactic violation. However, since one of the goals of the present study was to determine whether skipping a preview consisting of a syntactic violation occurs only for function word previews, it is important to check whether the effects we observed were driven entirely by such target and preview words. In order to do this, we performed analyses with an additional factor denoting whether both target and preview words were content words (coded as 0 ) or whether either the preview or target word was a function word (coded as 1, see Appendix for details); there were no cases in which both preview and target were function words. There was no significant interaction effect between preview and target/ preview word class on any of the skipping probabilities for the pre-target, target, and posttarget words and no significant three-way interaction between preview, sentence frame, and target/preview word class (all $z<1.96$ ), with one exception: the post-target word was skipped more often when the preview had been dissimilar (as opposed to alternate) when the target word was a function word $(b=.71, \mathrm{SE}=.25, z=2.8)$. Importantly, the effect we observed on the probability of skipping the target word did not differ depending on whether 
target and preview words were content or function words. There was only one significant interaction effect on fixation time measures. Specifically, there was a significant three-way interaction on go-past time on the target word between the identical vs. alternative preview contrast, the sentence frame, and target word class $(b=-130.11, \mathrm{SE}=60.08, t=-2.17)$, suggesting that the preview benefit effect on go-past time on the target word was only present for those sentence frames in which the target word was a high-frequency function word. None of these interactions provide evidence that detracts from our primary claim that high frequency parafoveal words are skipped more often than low frequency parafoveal words even if the sentence context favors the low frequency word. Furthermore, based on these analyses, it appears that the content or function status of a word did not modulate these effects.

Target word predictability—Finally, the target words differed in terms of their predictability from the preceding sentence context. While the majority of the target words were not predictable from the context, a number of target words were somewhat constrained by the sentence context. We therefore collected target word cloze data for all sentence frames from 11 UK native speakers in order to assess predictability. Predictability was low for most, but not all of the target words with a few exceptions (mean $=.07$; range $=0-.91$ ) In order to assess whether predictability modulated the effects we observed, we performed another set of analyses in which we included target word predictability in the analysis. Given that there were 11 subjects, we entered target word predictability into the model as a categorical rather than a continuous variable (target word or target word preview were predicted by at least one norming subject, coded as 1 -this included $29 \%$ of items with an average predictability of .23-vs. neither target word nor preview were predicted by any norming subjects, coded as 0 - this included $71 \%$ of items; see Appendix for details). There were no significant two- or three-way interaction effects of target word predictability on the probability of skipping the pre-target, target, or post-target word, suggesting that target word predictability did not affect subjects' skipping decisions in the present study (all z < 1.96). There were a few significant interaction effects on fixation time measures: On the pre-target word, there were significant three-way interactions between target word predictability, sentence frame, and each of the preview contrasts (interaction term for identical vs. alternative: $\mathrm{b}=-34.08, \mathrm{SE}=13.63, t=-2.5$; alternative vs. dissimilar: $\mathrm{b}=29.94, \mathrm{SE}=$ $13.36, t=2.24)$. This is quite interesting, as it suggests that there was a parafoveal-on-foveal effect of preview when the target word was predictable from the sentence context, but no parafoveal-on-foveal effect of preview when it was not predictable. Separate analyses for the sentences with predictable target words and those with non-predictable target words produced interactions between the preview contrasts and the sentence frame that were not significant for those sentences in which the target word was not predictable (all $t \mathrm{~s}<1$ ), but highly significant for those sentences in which it was. However, this effect was only significant for the sentence frames compatible with high-frequency target words (Interaction term for identical vs. alternative: $\mathrm{b}=-42.02, \mathrm{SE}=11.43, t=-3.68$; alternative vs. dissimilar: $\mathrm{b}=30.72, \mathrm{SE}=11.21, t=2.74)$. Therefore, it appears that subjects may have, at least in some cases, predicted the identity of the target word, and when the target preview was incompatible with the prediction, they experienced some disruption. A similar effect was observed by Rayner et al. (2013). Such a prediction mechanism could also explain the 
apparent parafoveal-on-foveal effects we observed for skipping the pre-target word, although this would suggest that both prediction generation and comparison of the prediction with the parafoveal preview can occur as much as two words ahead. Such a suggestion might occur if the perceptual span is extended when upcoming words are highly predictable. Finally, the interaction between the identical vs. alternative preview contrast and word predictability on FFD on the post-target word was significant $(b=-20.17, \mathrm{SE}=$ $9.54, t=2.12$ ), suggesting that, for predictable target words, there was no spillover effect of having had the alternative preview—on the contrary, FFDs on the post-target word were slightly lower in the alternative preview condition compared to the identical condition.

\section{General Discussion}

In the present experiment, we pitted two sources of information about the upcoming word against each other. In the critical preview condition, the information from the available parafoveal preview contradicted expectations about the upcoming word based on the syntactic structure of the preceding sentence. Angele and Rayner (2012) showed that, in cases when the preview indicates that the upcoming word is the article the, but the sentence context is incompatible with that conclusion, the article interpretation wins: readers skip the apparent article and incur substantial processing difficulties later on. The present experiment demonstrates that a similar effect occurs for other three-letter words: First, random letter non-words were skipped less often than words. Second, the frequency of the upcoming word, but not its fit with the sentence, determined whether it was skipped. This finding is also consistent with the findings of White (2008), who showed that high frequency words are usually skipped more often than low-frequency words.

The present findings suggest that, at least for very short words, lexical properties of the parafoveal preview have a substantial influence on fixation probability, while its compatibility with the preceding sentence context matters to a far lesser degree.

Additionally, properties of the preview affect later processing, as shown by higher regression probabilities and go-past times. Our findings also suggest that readers are able to obtain some lexical information about parafoveal words, which fits neatly with the fact that readers seem to be able to skip even difficult words and still comprehend a sentence without problems. On the other hand, while expectations based on the preceding sentence context certainly play a role in shaping reading behavior (see Bicknell \& Levy, 2011), parafoveal lexical processing seems to trump the sentence context when it comes to word skipping.

A remaining question is how our results relate to previous findings about the influence of predictability on word skipping (Balota et al., 1985; Drieghe, Rayner, \& Pollatsek, 2005; Ehrlich \& Rayner, 1983; Rayner \& Well, 1996; for a review see Brysbaert, Drieghe, \& Vitu, 2005), which suggest that readers are more likely to skip highly predictable words. Given this, why did we not see more pronounced effects of predictability in the present study? As noted above, this likely occurred because our target words were, in general, not strongly predictable (see Appendix for details). It also remains to be determined whether our results generalize to longer words (for a recent study on the skipping of longer words, see Choi \& Gordon, 2013). 
In summary, the present study demonstrates that skipping of short words is strongly influenced by the frequency of its parafoveal preview. This suggests that the the-skipping effects observed by O'Regan, (1979), Gautier et al. (2000), and Angele and Rayner (2012) are not necessarily specific to the definite article the, but rather, they occur as a function of its word frequency. On a more general level, our results underline the importance of parafoveal orthographic and lexical processing in comparison with higher-level processing such as syntactic or semantic processing. While syntactic integration and semantic processing occur during or after a word has been fixated, the information available about a word that is still in the parafovea is mainly of either an orthographic or lexical nature. Importantly, this does not mean that parafoveal information about a word is irrelevant once the eyes have moved - on the contrary, we found clear effects of the preview of a word having been dissimilar once that word is finally fixated and even on the subsequent fixations. Clearly, however, there is no evidence with this experimental paradigm that syntactic and semantic information about the upcoming word and its fit with the sentence affect the timing of the skipping decision.

\section{Implications for computational models}

Our findings show that readers routinely skip parafoveal high frequency words without taking the syntactic or semantic sentence context into account. This demonstrates that, at least with regards to skipping short words, the current implementations of both the E-Z Reader and SWIFT models are adequate: In both models, the time needed to parafoveally process a word (and its fixation probability) is strongly dependent on its frequency. Both models also predict that the processing time of a word should be influenced by its predictability, however, this is where our results do not correspond to the model predictions, as we saw no effect of fit with the sentence context. It is important to note, however, that both E-Z Reader 9 (Pollatsek, Reichle, \& Rayner, 2006), and SWIFT focus on word identification rather than semantic integration. As such, they both allow parafoveal identification of an upcoming word prior to skipping it. Version 10 of E-Z Reader (Reichle et al., 2009) goes further than this by including a processing stage representing the syntactic and semantic integration of a word into the sentence context. If one assumes that the integration stage in E-Z Reader 10 would necessarily fail to integrate the infelicitous the preview into the sentence, E-Z Reader 10 might be able to explain why there was disruption only subsequent to skipping the infelicitous the preview. Keeping in mind that there are previous results supporting an effect of predictability on word skipping and that our experiment really only measures the difference in skipping between an unpredictable and grammatically illegal (or, in the case of the semantic violations, at least implausible, if not anomalous) parafoveal word, some changes in how the models treat the predictability of parafoveal words might be necessary. However, such changes would be rather small modifications of existing mechanisms in both models and would not require that new mechanisms be proposed. Furthermore, these effects can be accounted for by both parallel and serial accounts of word identification.

The unexpected parafoveal-on-foveal effect of the preview condition on skipping probability and fixation time on the pre-target word might have stronger implications for the models. If there is indeed an early parafoveal plausibility check, such a mechanism is not present in 
either model and would have to be added. One might argue that processing far ahead of the fixated word may be more in the "spirit" of parallel processing, however, it would not be impossible to conceive of a serial model that involves a very early parallel visual stage in which all letters in the perceptual span are superficially processed at the same time (in fact, the "V" stage in E-Z Reader is described as exactly such a process, though this is not implemented in current versions of the model). This superficial processing could involve checking whether parafoveal word information is consistent with those words that are preactivated from processing the context. It is important to note at this point that, in order to warrant model modifications, the parafoveal-on-foveal effects described above will have to be replicated in a dedicated experiment. They do suggest some interesting directions for future research, however.

In summary, our finding that high frequency parafoveal words are often skipped without taking the sentence context into account is, in principle, compatible with both E-Z Reader and SWIFT (pending minor modifications). Explaining our findings on the pre-target word might require more fundamental model changes, but further research is required to replicate those effects in a controlled experiment. On a more general level, our results indicate that there is at least one important process during reading which does not seem to be affected by word predictability from the context, but just by the properties of the parafoveal word. This may pose a problem to models, such as that of Bicknell and Levy (2010), that propose that readers constantly evaluate the available evidence about previously fixated, currently fixated, and upcoming words since such an evaluation should alert readers to the mismatch between prediction and actual parafoveal information. While we did find some evidence for such a parafoveal plausibility check, the effects we observed were not strong enough to warrant the assumption that such a check is carried out during every fixation. Rather, it seems that, during normal reading, parafoveal plausibility is only checked once in a while if at all.

\section{Acknowledgments}

This research was supported by Leverhulme Trust Grant F/00 180/AN, Economic and Social Research Council Grant ES/I032398/1 and by National Institutes of Health Grant HD26765. We thank Emily Morgan and Roger Levy for their help with collecting the cloze norming data, and Reinhold Kliegl for comments on a prior draft.

\section{References}

Angele B, Rayner K. Processing the in the parafovea: Are articles skipped automatically? Journal of Experimental Psychology: Learning, Memory, and Cognition. 2013; 39(2):649-662.

Baayen RH, Davidson DJ, Bates DM. Mixed-effects modeling with crossed random effects for subjects and items. Journal of Memory and Language. 2008; 59:390-412.

Balota DA, Pollatsek A, Rayner K. The interaction of contextual constraints and parafoveal visual information in reading. Cognitive Psychology. 1985; 17:364-390. [PubMed: 4053565]

Bates, D.; Maechler, M.; Bolker, B.; Walker, S. lme4: Linear mixed-effects models using Eigen and S4. R package version 1.0-5. 2013. http://CRAN.R-project.org/package=lme4

Bicknell K, Levy R. A rational model of eye movement control in reading. Proceedings of the 48th Annual Meeting of the Association for Computational Linguistics. 2010:1168-1178.

Bicknell, K.; Levy, R. Why readers regress to previous words: A statistical analysis. In: Carlson, L.; Hölscher, C.; Shipley, T., editors. Proceedings of the 33rd Annual Conference of the Cognitive Science Society. Austin, TX: Cognitive Science Society; 2011. p. 931-936. 
Brysbaert, M.; Drieghe, D.; Vitu, F. Word skipping: Implications for theories of eye movement control in reading. In: Underwood, G., editor. Cognitive processes in eye guidance. Oxford: Oxford University Press; 2005. p. 53-77.

Choi W, Gordon PC. Coordination of word recognition and oculomotor control during reading: the role of implicit lexical decisions. Journal of Experimental Psychology: Human Perception and Performance. 2013; 39(4):1032-1046. [PubMed: 23106372]

Drieghe D, Rayner K, Pollatsek A. Eye movements and word skipping during reading revisited. Journal of Experimental Psychology: Human Perception and Performance. 2005; 31:954-969. [PubMed: 16262491]

Davis CJ. N-watch: A program for deriving neighborhood size and other psycholinguistic statistics. Behavior Research Methods. 2005; 37(1):65-70. [PubMed: 16097345]

Ehrlich SF, Rayner K. Contextual effects on word perception and eye movements during reading. Journal of Verbal Learning and Verbal Behavior. 1981; 20:641-655.

Engbert R, Nuthmann A, Richter EM, Kliegl R. SWIFT: A dynamical model of saccade generation during reading. Psychological Review. 2005; 112:777-813. [PubMed: 16262468]

Gautier V, O'Regan JK, Le Gargasson JF. “The-skipping” revisited in French: programming saccades to skip the article "les". Vision Research. 2000; 40:2517-2531. [PubMed: 10915890]

Hautala J, Hyönä J, Aro M. Dissociating spatial and letter-based word length effects observed in readers' eye movement patterns. Vision Research. 2011; 51:1719-1727. [PubMed: 21664920]

Kennedy, A. The influence of parafoveal words on foveal inspection time: Evidence for a processing trade-off. In: Underwood, G., editor. Eye Guidance in Reading and Scene Perception. Oxford, UK: Elsevier; 1998. p. 149-179.

Kennedy A, Murray W, Boissiere C. Parafoveal pragmatics revisited. European Journal of Cognitive Psychology. 2004; 16:128-153.

Kennedy A, Pynte J. Parafoveal-on-foveal effects in normal reading. Vision Research. 2005; 45:153168. [PubMed: 15581917]

Kennedy A, Pynte J, Ducrot S. Parafoveal-on-foveal interactions in word recognition. The Quarterly Journal of Experimental Psychology Section A. 2002; 55:1307-1337.

Kliegl R, Nuthmann A, Engbert R. Tracking the mind during reading: The influence of past, present, and future words on fixation durations. Journal of Experimental Psychology-General. 2006; 135:12-35. [PubMed: 16478314]

Levy R, Bicknell K, Slattery T, Rayner K. Eye movement evidence that readers maintain and act on uncertainty about past linguistic input. Proceedings of the National Academy of Sciences. 2009; 106:21086-21090.

Liversedge SP, Findlay JM. Saccadic eye movements and cognition. Trends in Cognitive Sciences. 2000; 4:6-14. [PubMed: 10637617]

McConkie GW, Rayner K. The span of the effective stimulus during a fixation in reading. Perception \& Psychophysics. 1975; 17:578-587.

O'Regan K. Saccade size control in reading: Evidence for the linguistic control hypothesis. Perception \& Psychophysics. 1979; 25:501-509. [PubMed: 492916]

Pollatsek A, Reichle ED, Rayner K. Tests of the E-Z Reader model: Exploring the interface between cognition and eye-movement control. Cognitive Psychology. 2006; 52:1-56. [PubMed: 16289074]

R Development Core Team, R. R: a language and environment for statistical computing. R Foundation for Statistical Computing. 2013

Rayner K. The perceptual span and peripheral cues in reading. Cognitive Psychology. 1975; 7:65-81.

Rayner K. Eye movements in reading and information processing: 20 years of research. Psychological Bulletin. 1998; 124:372-422. [PubMed: 9849112]

Rayner K. The Thirty-Fifth Sir Frederick Bartlett Lecture: Eye movements and attention in reading, scene perception, and visual search. The Quarterly Journal of Experimental Psychology. 2009; 62:1457-1506. [PubMed: 19449261]

Rayner K, Angele B, Schotter ER, Bicknell K. On the processing of canonical word order during eye fixations in reading: Do readers process transposed word previews? Visual Cognition. 2013; 21(3): 353-381. [PubMed: 24003322] 
Rayner, K.; Liversedge, S. Linguistic and cognitive influences on eye movements during reading. In: Liversedge, S.; Gilchrist, I.; Everling, S., editors. The Oxford Handbook of Eye Movements. Oxford, UK: Oxford University Press; 2011.

Rayner K, McConkie GW. What guides a reader's eye movements? Vision Research. 1976; 16:829_ 837. [PubMed: 960610]

Rayner K, Well AD. Effects of contextual constraint on eye movements in reading: A further examination. Psychonomic Bulletin \& Review. 1996; 3:504-509. [PubMed: 24213985]

Reichle ED, Pollatsek A, Fisher DL, Rayner K. Toward a model of eye movement control in reading. Psychological Review. 1998; 105:125-157. [PubMed: 9450374]

Reichle ED, Warren T, McConnell K. Using E-Z Reader to model the effects of higher level language processing on eye movements during reading. Psychonomic Bulletin \& Review. 2009; 16:1-21. [PubMed: 19145006]

Schotter ER, Angele B, Rayner K. Parafoveal processing in reading. Attention, Perception, \& Psychophysics. 2012; 74:5-35.

Slattery TJ, Angele B, Rayner K. Eye movements and display change detection during reading. Journal of Experimental Psychology. Human Perception and Performance. 2011; 37:1924-1938. [PubMed: 21688934]

Vitu F, O'Regan JK, Inhoff AW, Topolski R. Mindless reading: Eye-movement characteristics are similar in scanning letter strings and reading texts. Perception \& Psychophysics. 1995; 57(3):352364. [PubMed: 7770326]

White SJ. Eye movement control during reading: Effects of word frequency and orthographic familiarity. Journal of Experimental Psychology: Human Perception and Performance. 2008; 34:205-223. [PubMed: 18248149]

White S, Rayner K, Liversedge S. Eye movements and the modulation of parafoveal processing by foveal processing difficulty: A reexamination. Psychonomic Bulletin \& Review. 2005; 12:891896. [PubMed: 16524007]

\section{Appendix}




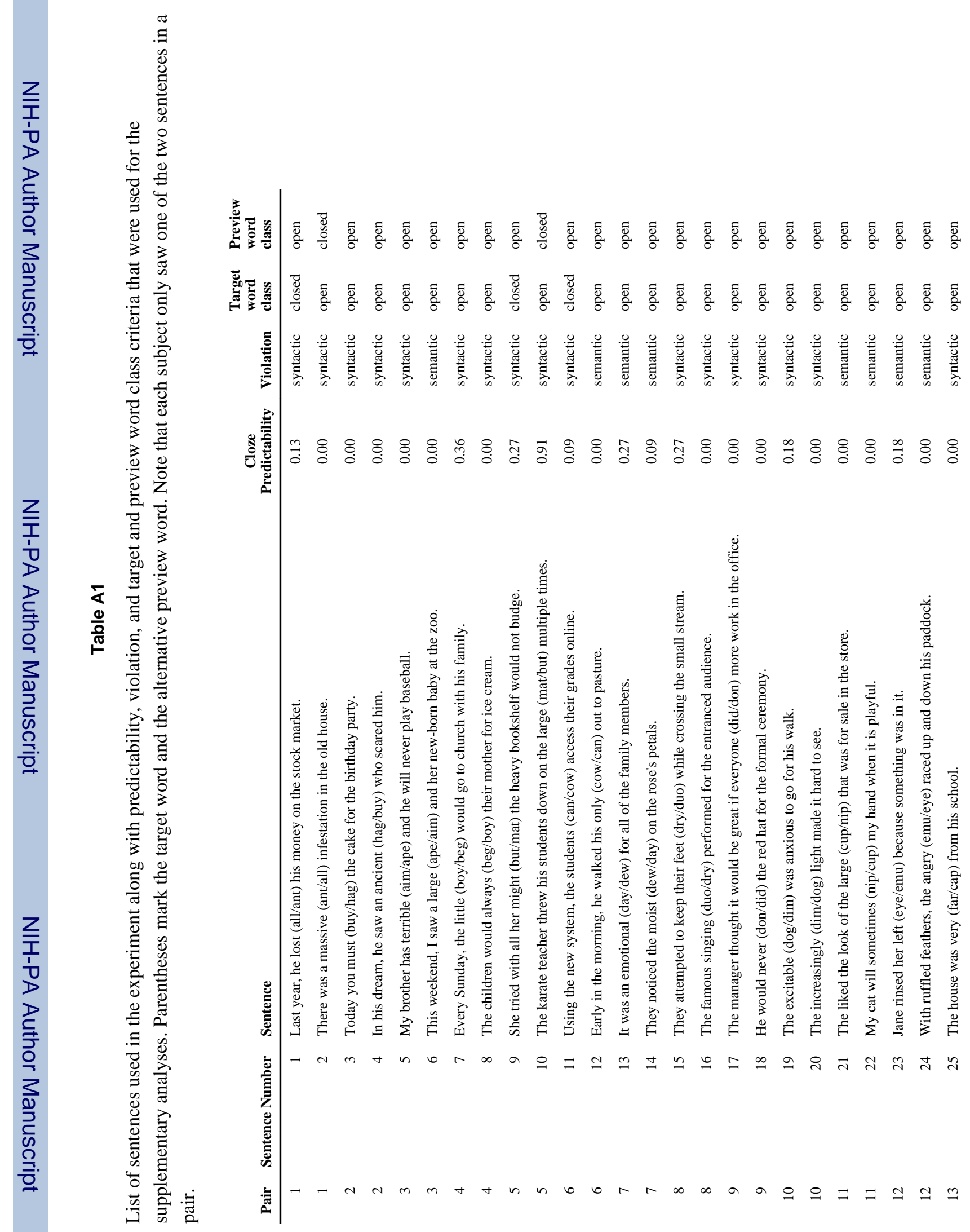

J Exp Psychol Learn Mem Cogn. Author manuscript; available in PMC 2015 July 01. 


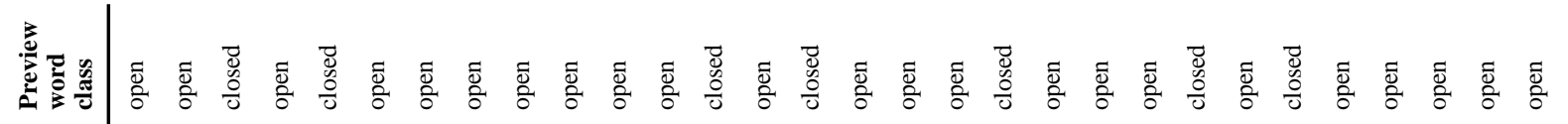

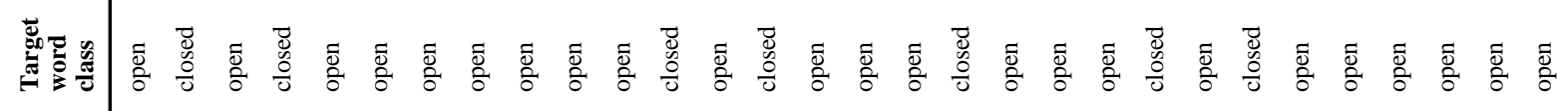

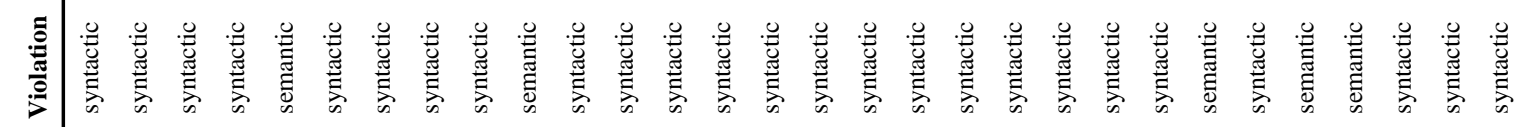

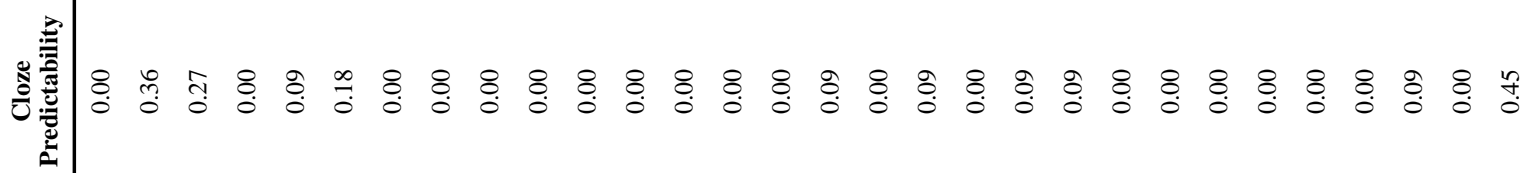

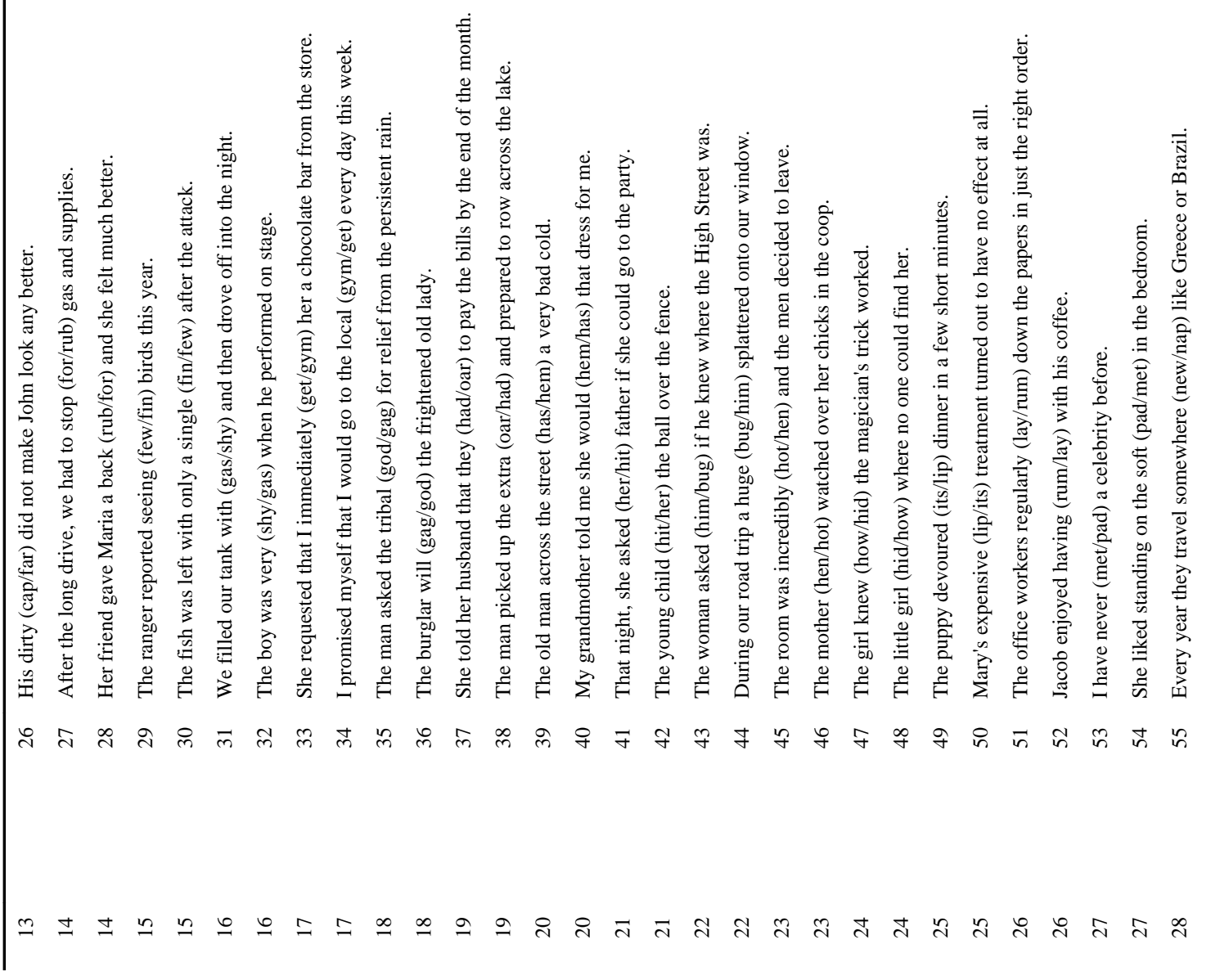




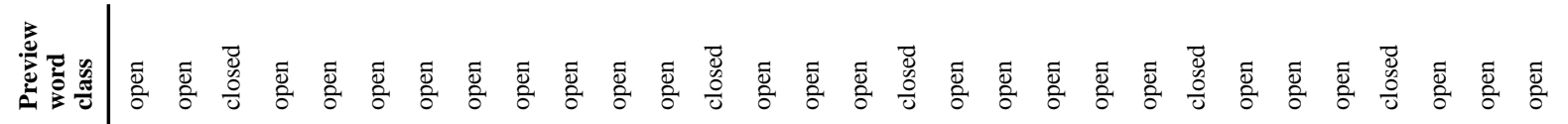

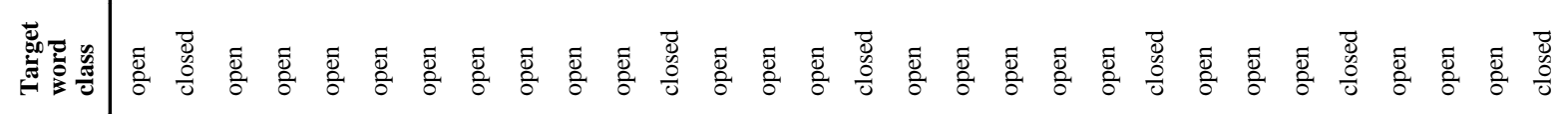

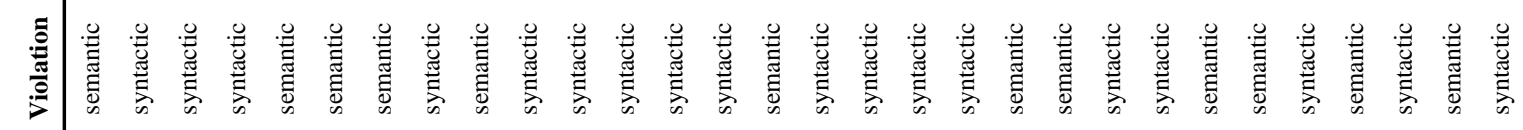




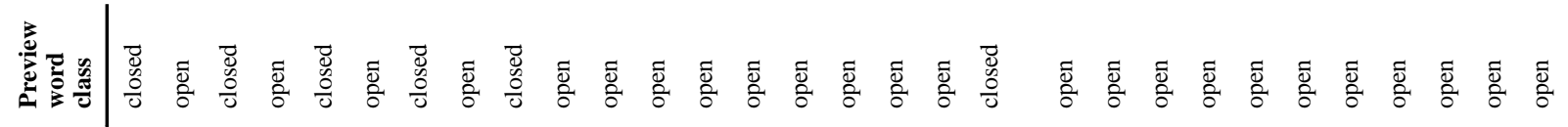

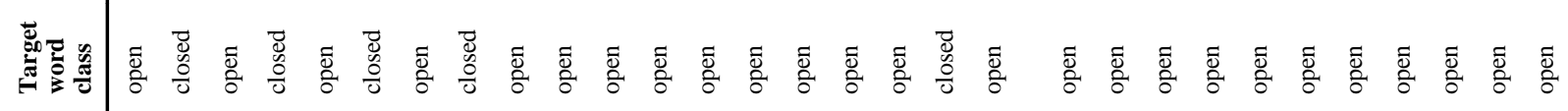

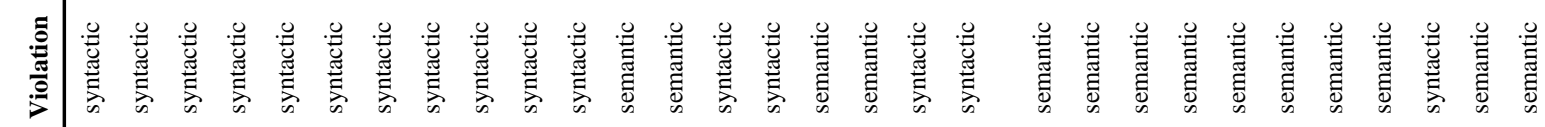

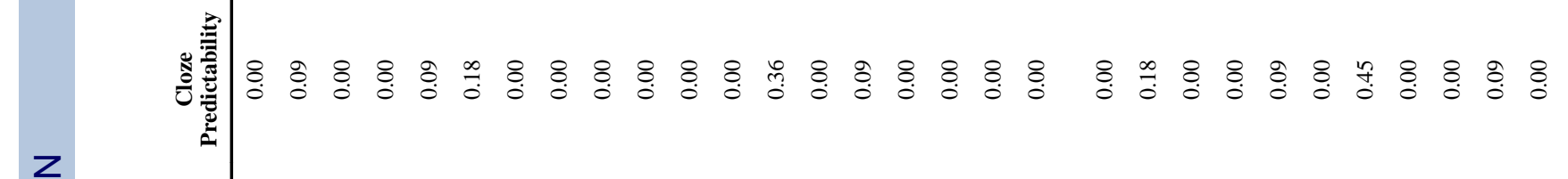




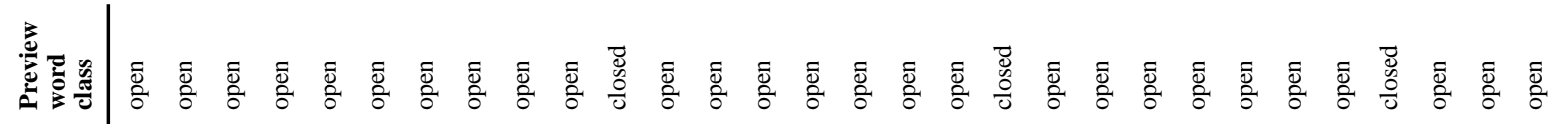

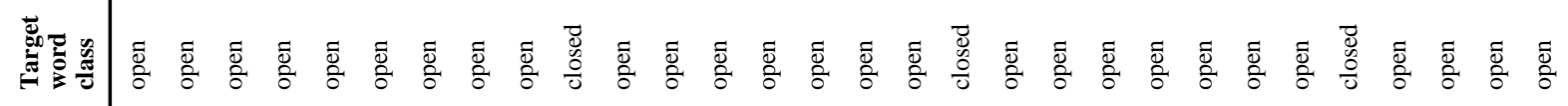

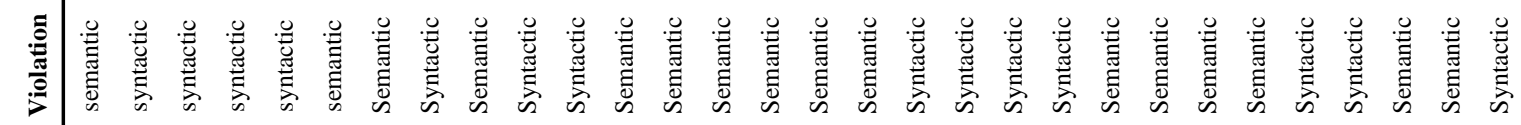




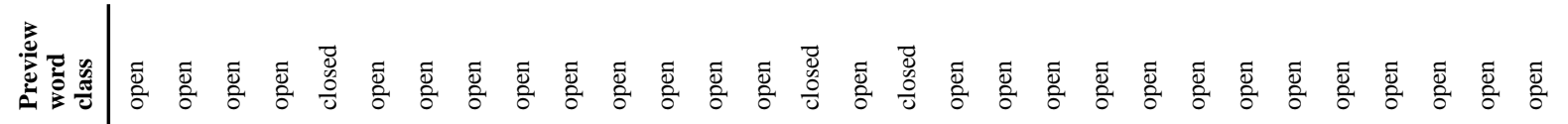

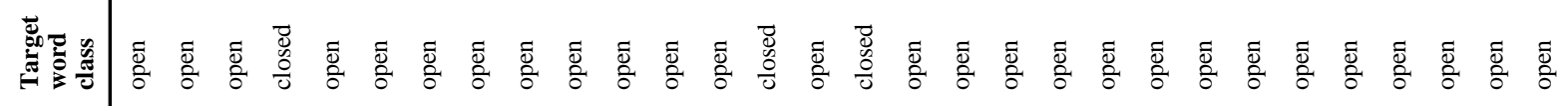

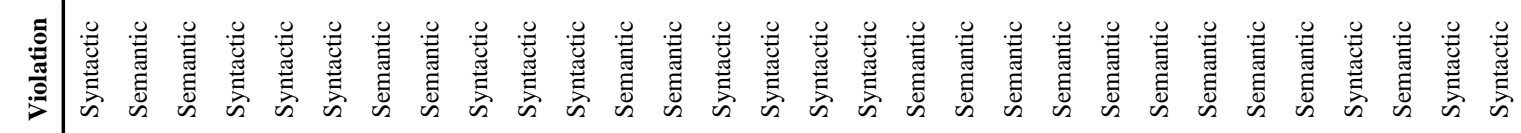

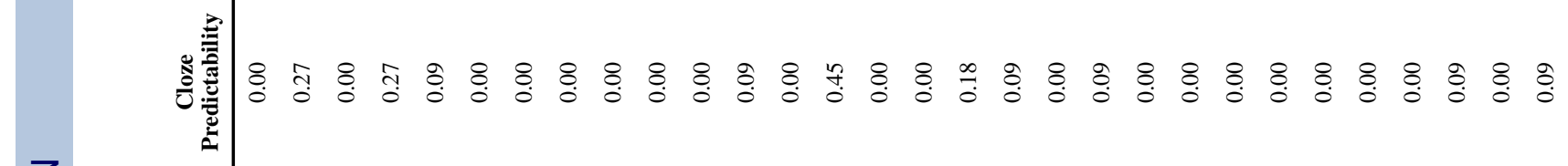

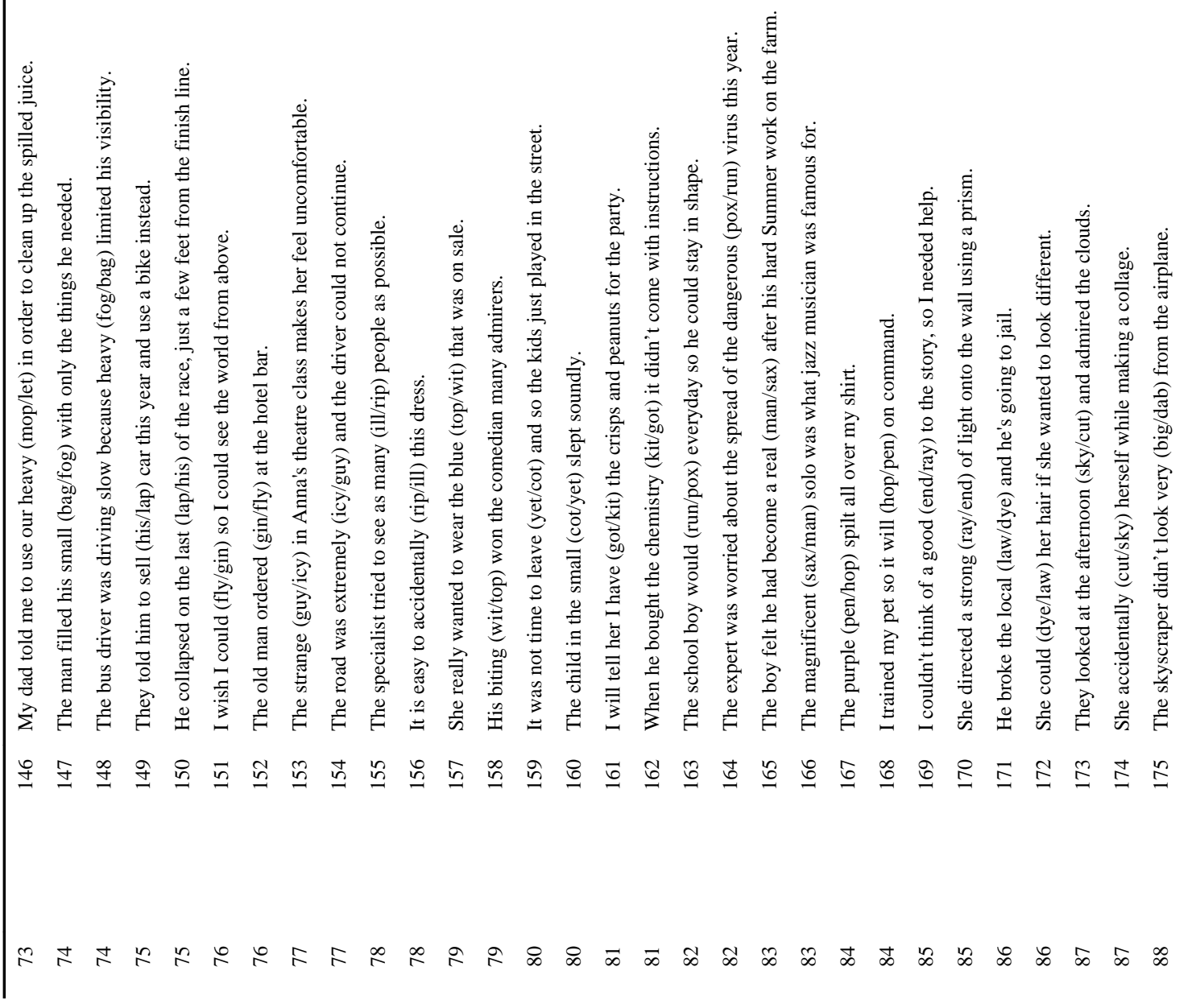

J Exp Psychol Learn Mem Cogn. Author manuscript; available in PMC 2015 July 01. 


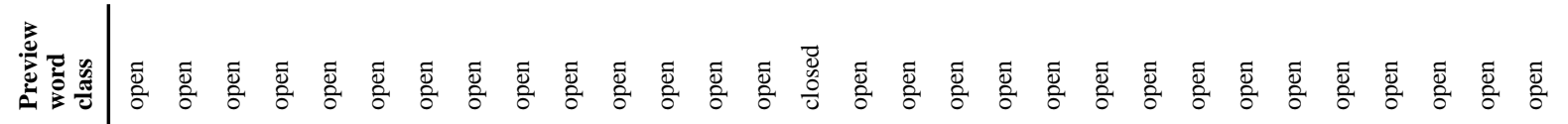

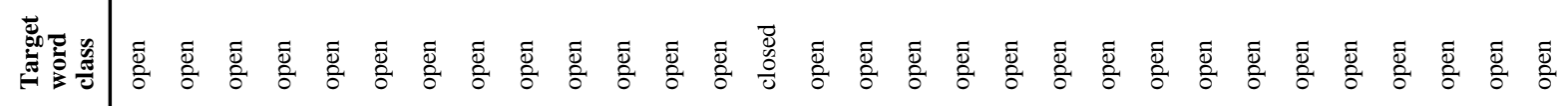

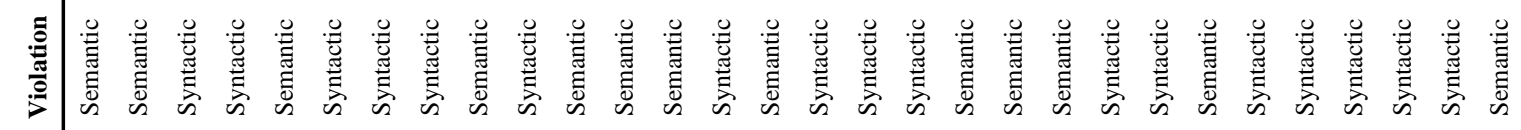

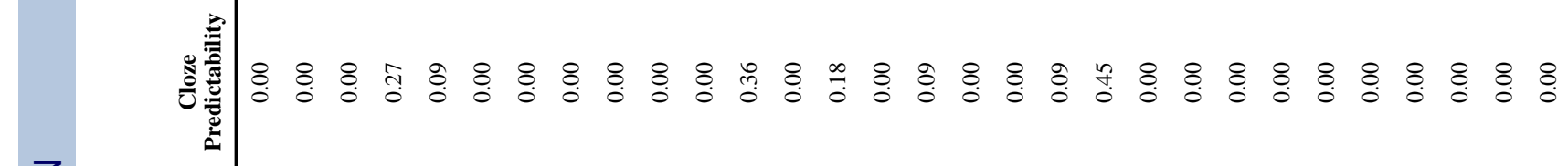

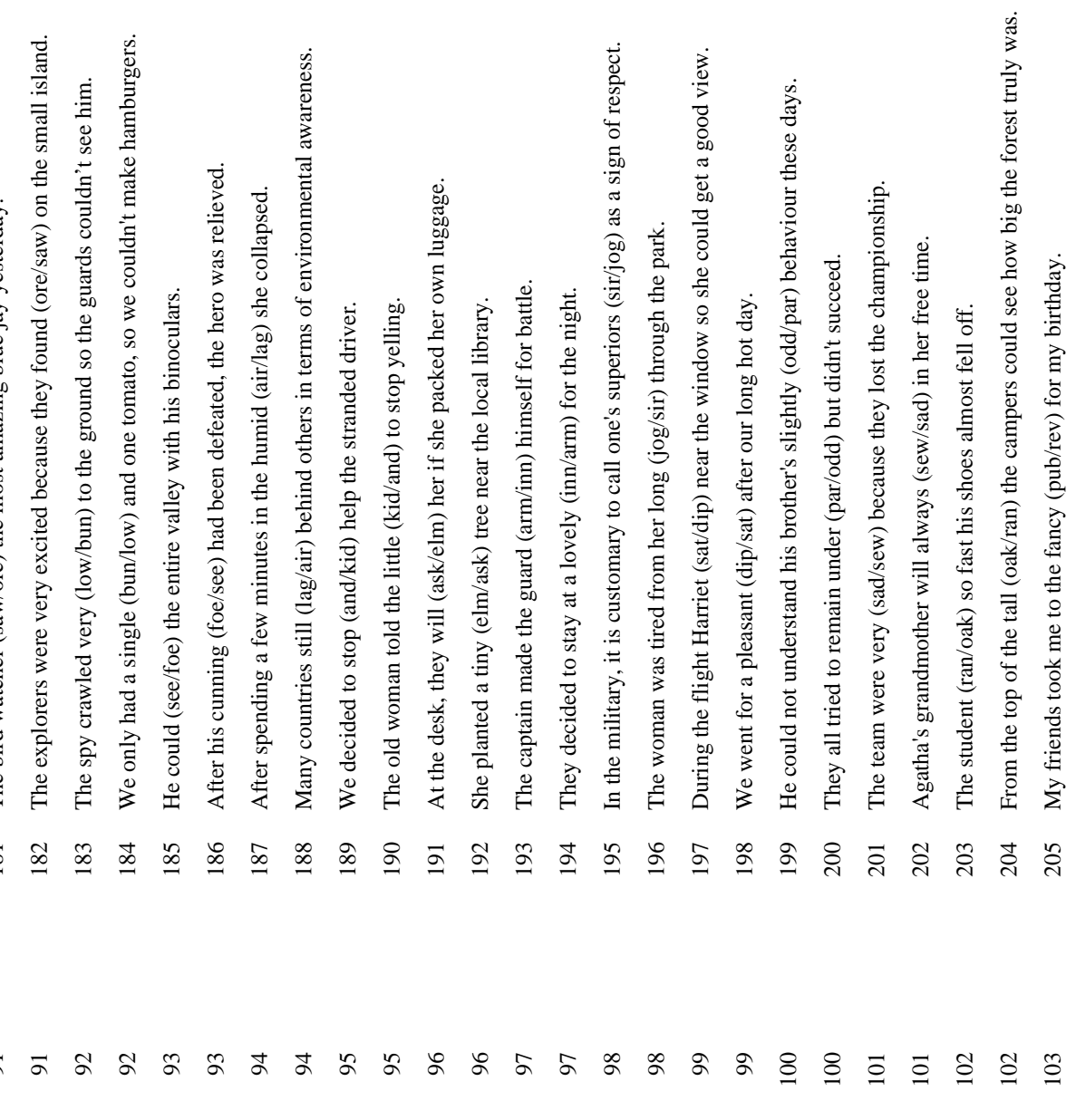




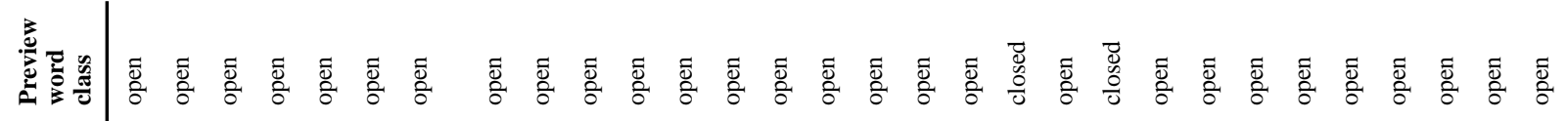

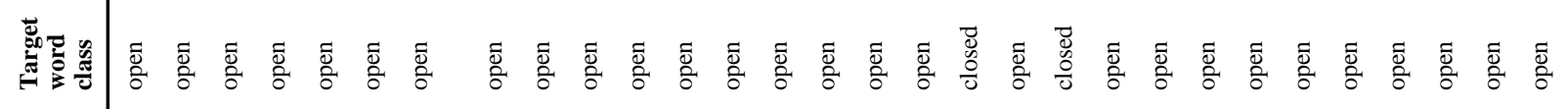

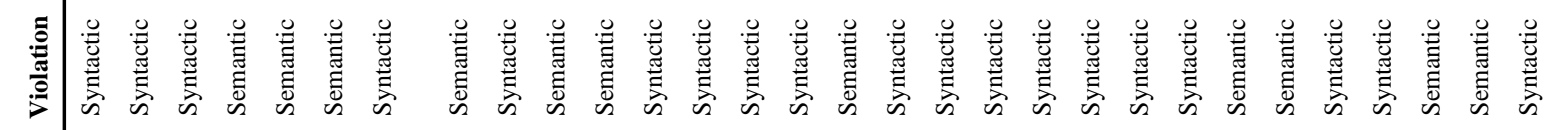

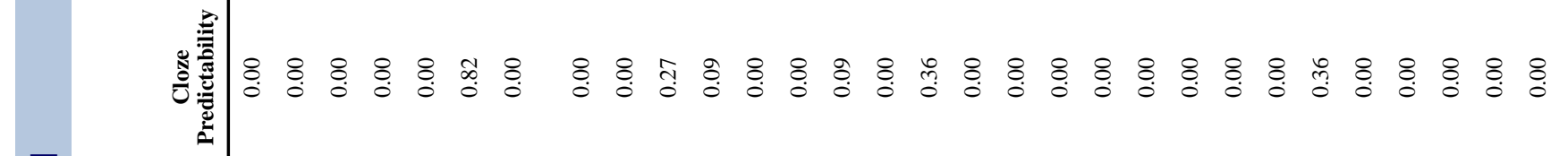




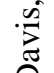

$\stackrel{0}{a}$ 


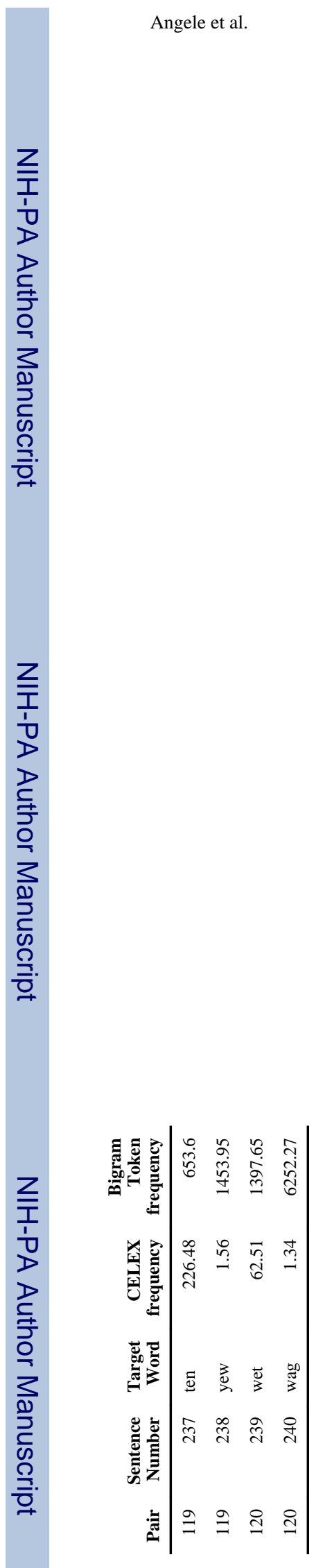

J Exp Psychol Learn Mem Cogn. Author manuscript; available in PMC 2015 July 01. 


\begin{tabular}{|c|c|c|c|}
\hline Sentence frame & Preview & Example sentence before display change & Example sentence after display change \\
\hline \multirow{3}{*}{$\begin{array}{l}\text { High-frequency } \\
\text { target }\end{array}$} & High-frequency & The excitable $e_{1}^{1}$ dog was eager to go for his walk. & \multirow{3}{*}{ The excitable dog was eager to go for his walk. } \\
\hline & Low-frequency & The excitable, dim was eager to go for his walk. & \\
\hline & Random letters & The excitable, hev was eager to go for his walk. & \\
\hline \multirow{3}{*}{$\begin{array}{l}\text { Low-frequency } \\
\text { target }\end{array}$} & High-frequency & The increasingly $y_{1}^{\prime}$ dog light made it hard to see. & \multirow{3}{*}{ The increasingly dim light made it hard to see. } \\
\hline & Low-frequency & The increasingly dim light made it hard to see. & \\
\hline & Random letters & The increasingly bup light made it hard to see. & \\
\hline
\end{tabular}

\section{Figure 1.}

Example stimuli from the present study. While readers were fixating to the left of the invisible boundary (dashed lined), the target word in each of the sentence frames (dog/dim) was replaced with a preview as shown in the left column. After a reader crossed the boundary, the preview was replaced with the actual target word (right column). 


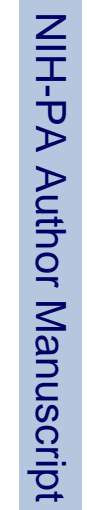

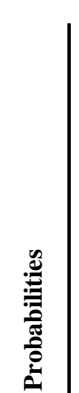

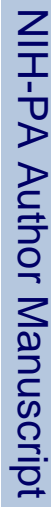

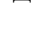

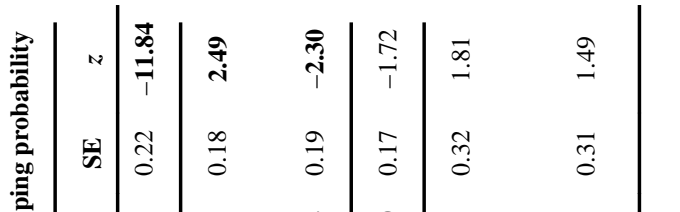

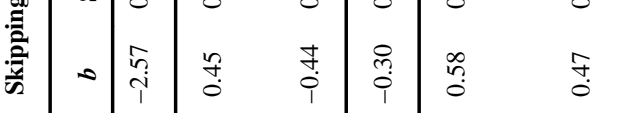

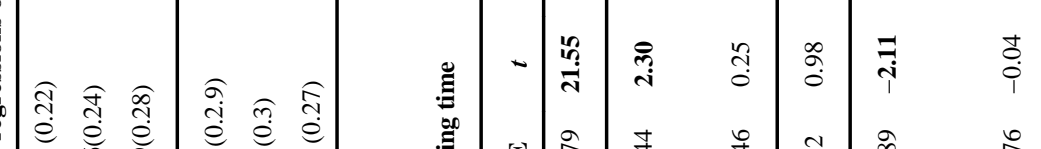

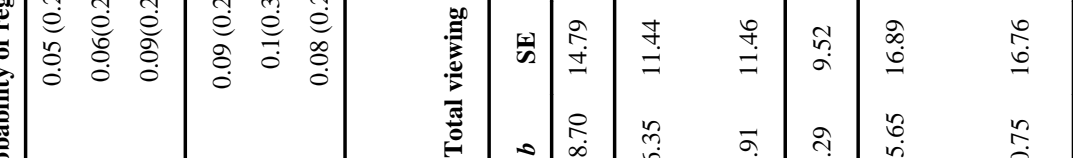

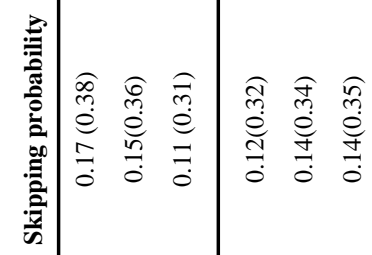

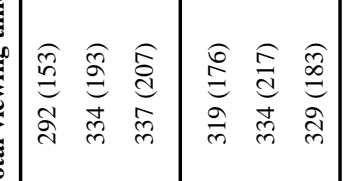

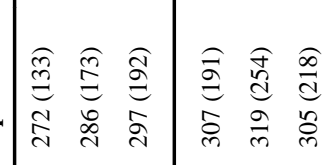

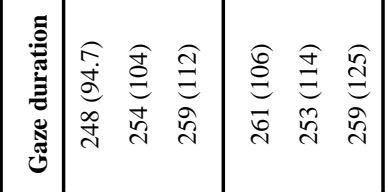

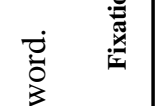

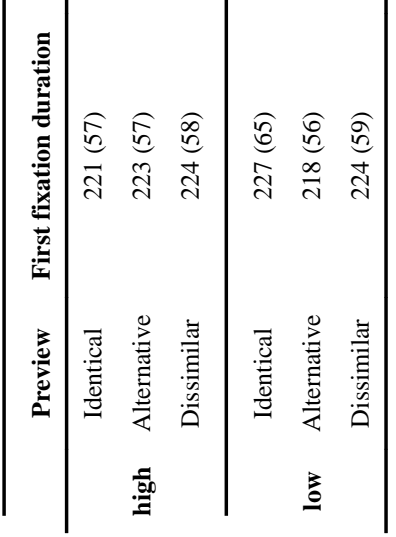

苛

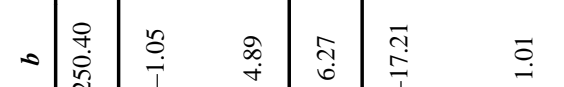

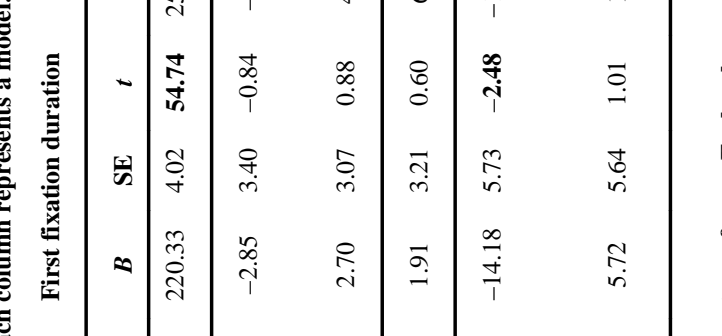

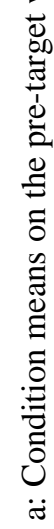

$\infty$

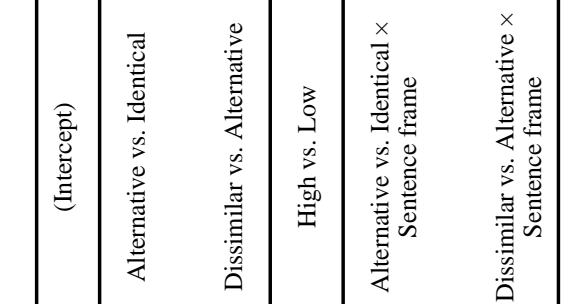

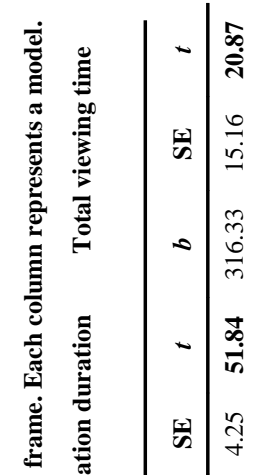

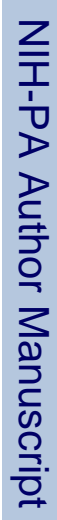

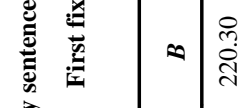

窇言

苞

要要 


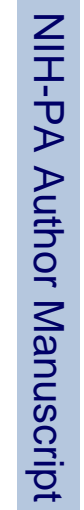

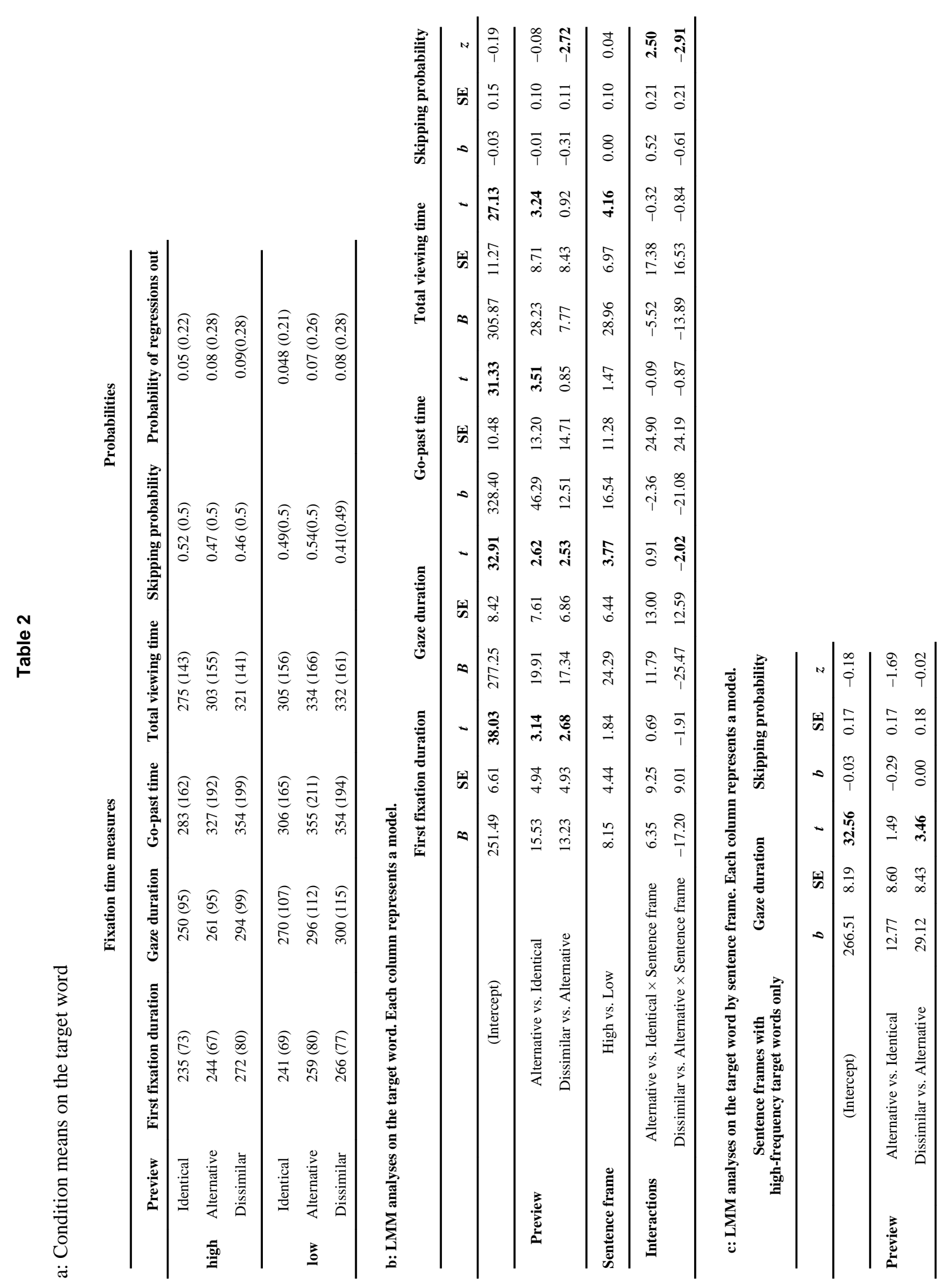

J Exp Psychol Learn Mem Cogn. Author manuscript; available in PMC 2015 July 01. 


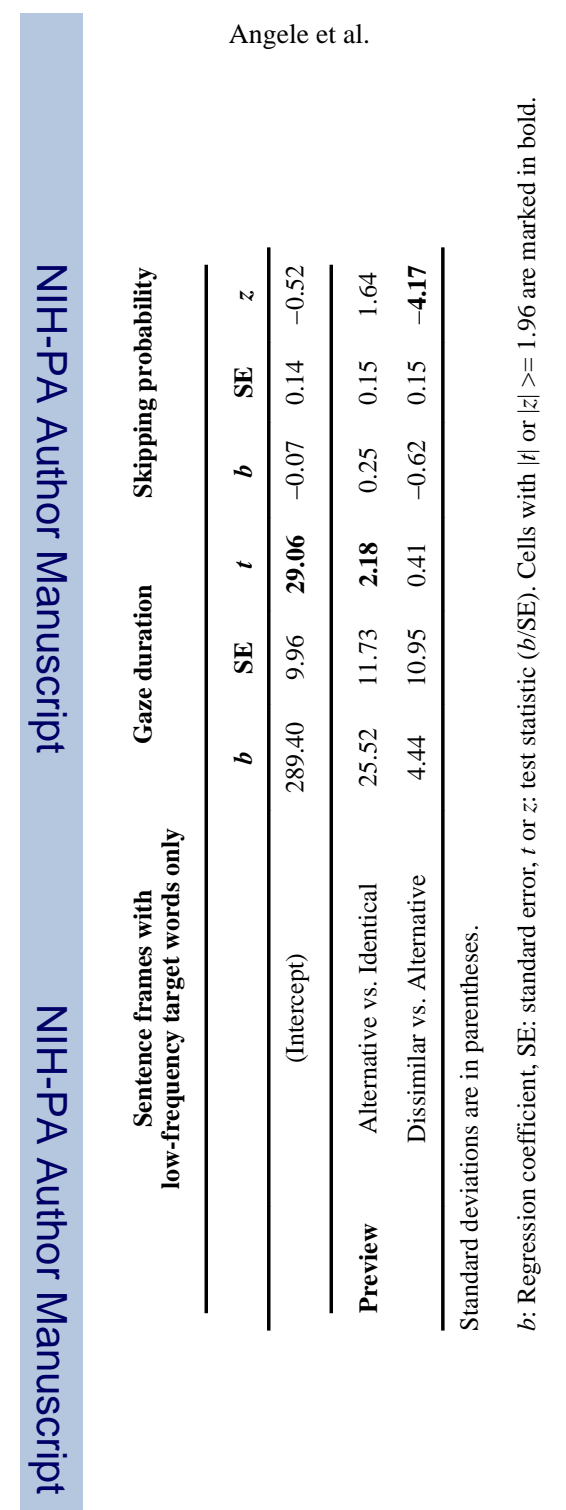

Page 39 


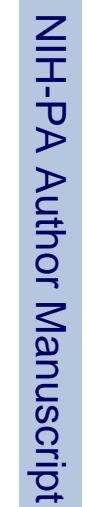

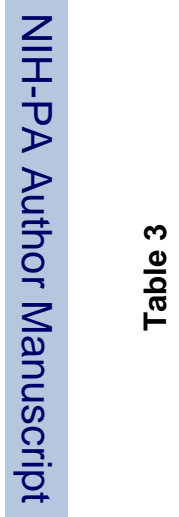

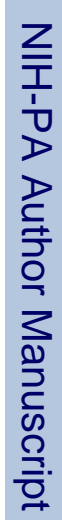

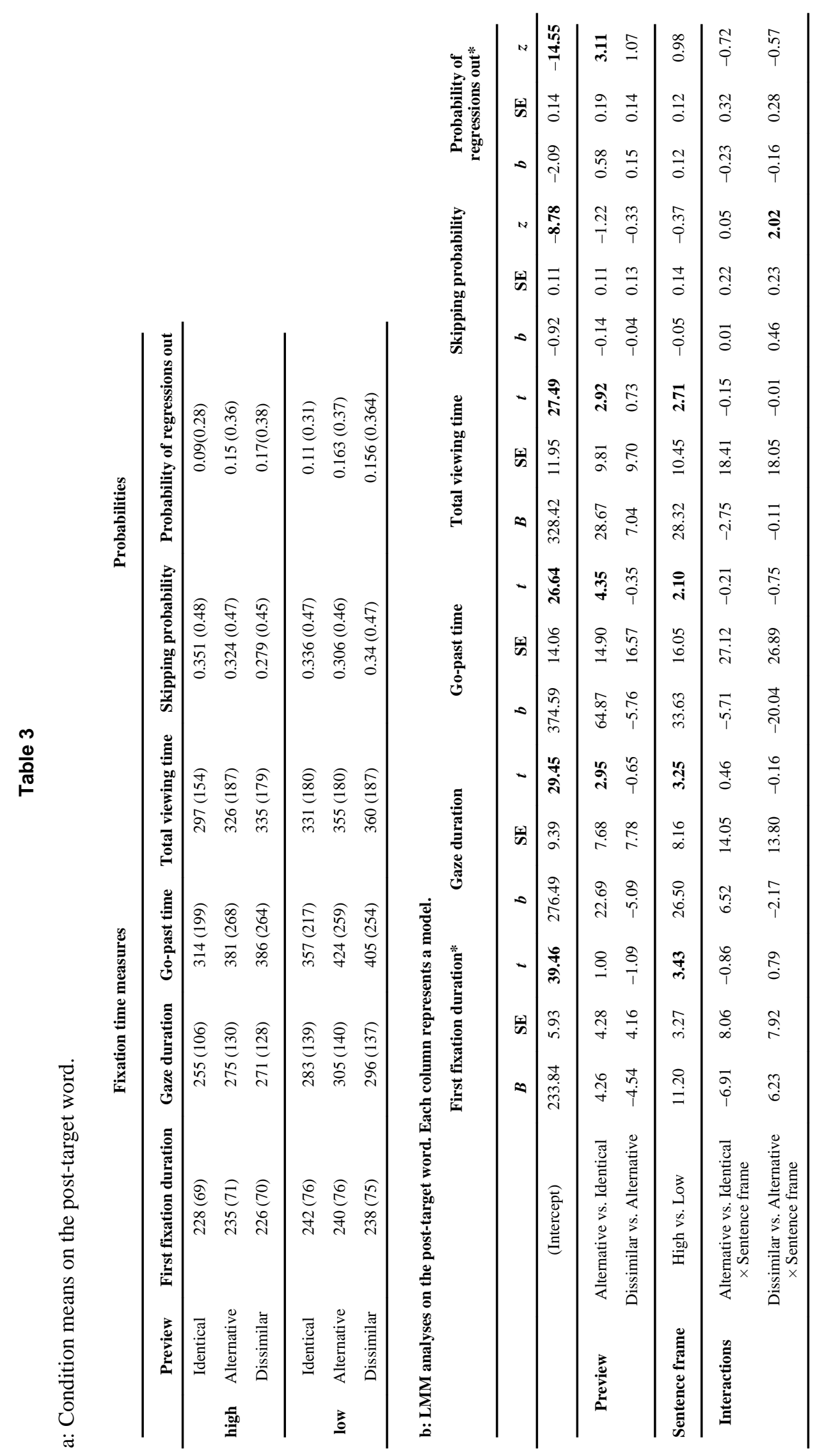

J Exp Psychol Learn Mem Cogn. Author manuscript; available in PMC 2015 July 01.

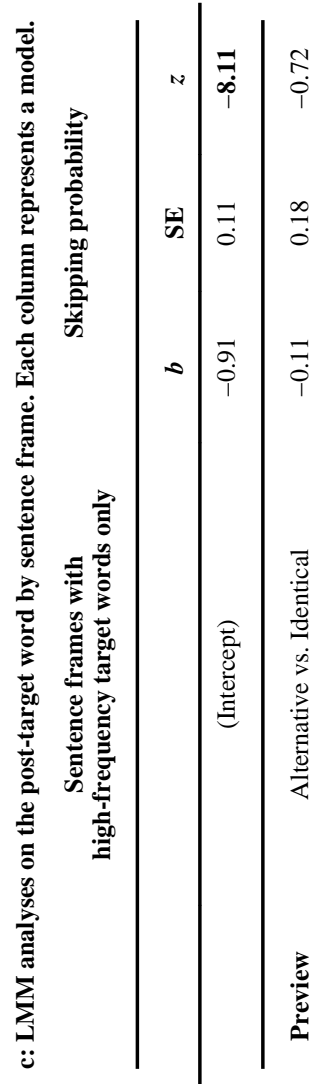


Angele et al.

Page 41

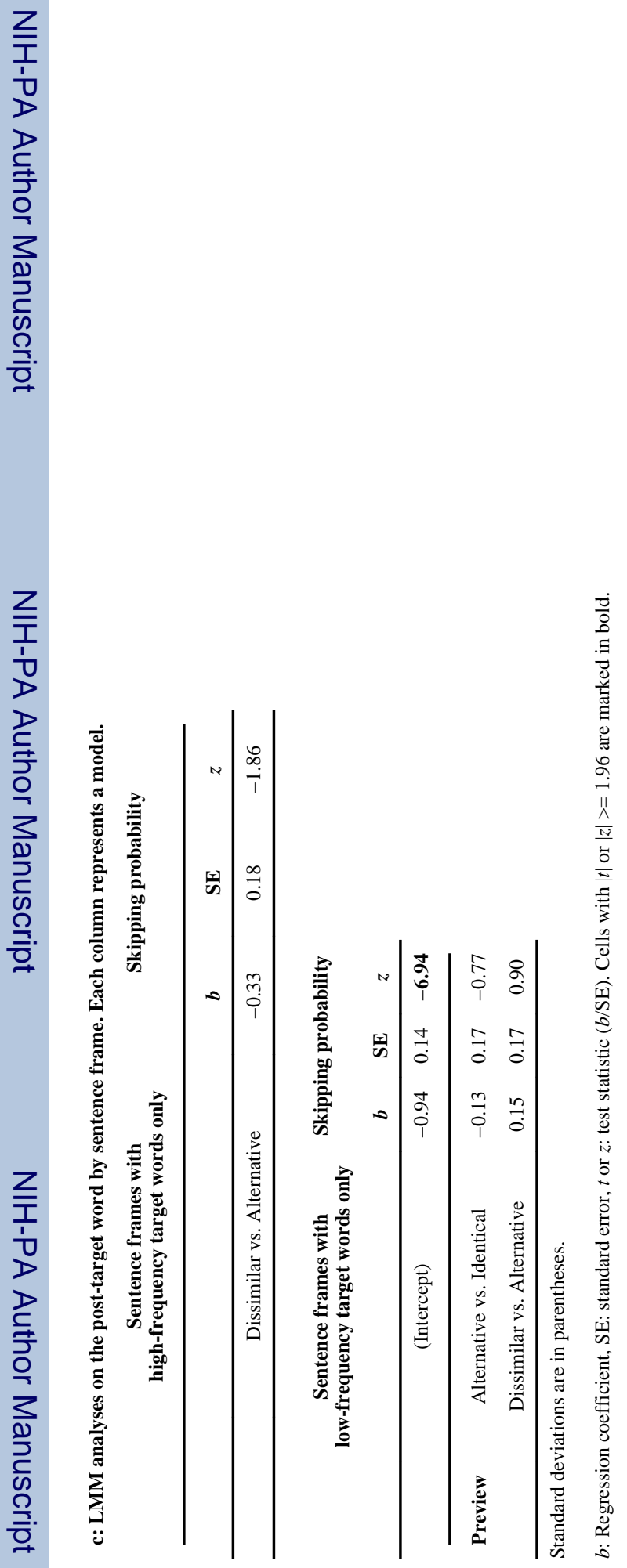

J Exp Psychol Learn Mem Conn. Author manuscript; available in PMC 2015 July 01. 